\title{
Intracardiac ultrasound two-dimensional and three-dimensional reconstruction for navigating percutaneous left atrial appendage occlusion
}

\author{
Witold Streb $^{1,2}{ }^{\circledR}$, Katarzyna Mitregga ${ }^{1}{ }^{1}$, Tomasz Podolecki ${ }^{1}$, Stanisław Morawski ${ }^{1}$, \\ Wiktoria Kowalska ${ }^{1}$, Wirginia Michlicka ${ }^{1}$, Zbigniew Kalarus ${ }^{1,2}$ (D) \\ ${ }^{1} 1^{\text {st }}$ Department of Cardiology and Angiology, Silesian Center for Heart Diseases in Zabrze, Poland \\ ${ }^{2}$ Department of Cardiology, Congenital Heart Diseases and Electrotherapy, \\ Silesian Medical University in Katowice, Poland
}

A growing portfolio of transcatheter procedures for structural heart disease poses new challenges in imaging techniques. Left atrial appendage closure (LAAC) represents a group of transcatheter procedures in which imaging is essential, both for guiding the procedure and as a tool for choosing the right occluder size [1-3]. Transesophageal echocardiography (TEE) has been the primary method of peri-procedural imaging, but new solutions are being sought to overcome its limitations. Evaluation is subject to intracardiac echocardiography (ICE) and methods that allow the fusion of images [4]. Only the use of ICE probes offering two-dimensional (2D) images during LAAC procedures has been evaluated so far. The current study compares the effectiveness of guiding the LAAC procedures employing the ICE 2D and ICE enabling three-dimensional reconstruction (ICE 3D).

The single-center prospective registry of LAAC procedures, included 330 consecutive patients with atrial fibrillation and contraindications for oral anticoagulant drugs, were analyzed. The preliminary selection included only LAAC procedures guided by both ICE $2 \mathrm{D}$ or ICE 3D. In the population of 31 patients separated in this way, LAAC procedures were performed using Amplatzer Amulet occluder. In 24 patients, LAAC was guided by the AccuNav $8 \mathrm{~F}$ probe enabling only $2 \mathrm{D}$ images, and in the remaining 7 cases, by the SoundStar $10 \mathrm{~F}$ probe, which enables 3D reconstruction. The left atrial appendage (LAA) was imaged by placing the AccuNav probe into the left atrium; for SoundStar probes, LAA images were captured from both the right and the left atrium in different atrial sections. Then the CartoSaund system was used for spatial reconstruction of LAA. All patients fulfilled the Amulet stability criteria before the device's release, as assessed by both ICE and fluoroscopy.

After 6 weeks, TEE was performed to exclude the leakage around the occluder and to assess if the implantation effect was optimal (the entire entrance to LAA covered with a disk without leaving any pouch, occluder axis parallel to LAA neck, separation between the device disk and lobe, optimal compression of the device). Whether ICE 2D or ICE 3D guided the treatment was blinded for the echocardiographer performing TEE.

Considering that the course and effects of LAAC procedures are influenced mainly by conditions related to the LAA anatomy and location of LAA orifice, the assessment of the results obtained with ICE 2D and ICE 3D was made based on cases with similar features. For this purpose, the propensity matching score technique was used. The following variables likely to impact the LAAC procedure were considered: the maximal dimension of the landing zone, the depth of LAA, the morphological type of LAA and the position of the LAA orifice in relation to the pulmonary veins. The matching was based on the optimal algorithm method using Euclidean distances and was carried

Address for correspondence: Dr. Witold Streb, Silesian Center for Heart Diseases in Zabrze, ul. Curie-Skłodowskiej 9, 41-800 Zabrze, Poland, tel: +48 3227134 14, e-mail: w.streb@sccs.pl

Received: $30.05 .2021 \quad$ Accepted: 28.08.2021 Early publication date: 10.09.2021

This article is available in open access under Creative Common Attribution-Non-Commercial-No Derivatives 4.0 International (CC BY-NC-ND 4.0) license, allowing to download articles and share them with others as long as they credit the authors and the publisher, but without permission to change them in any way or use them commercially. 
Table 1. Summary of matched intracardiac echocardiography (ICE) two-dimensional (ICE 2D) and ICE three-dimensional (ICE 3D) group characteristics.

\begin{tabular}{lcccc}
\hline Variable & Total & 3D ICE & 2D ICE & P level \\
\hline LA [mm] & $41.64 \pm 3.93$ & $40.29 \pm 3.99$ & $43.00 \pm 3.65$ & 0.118 \\
LVEDD [mm] & $49.6 \pm 7.967$ & $46.71 \pm 9.32$ & $52.57 \pm 5.53$ & 0.159 \\
LVESD [mm] & $37.36 \pm 9.68$ & $34.29 \pm 10.83$ & $40.43 \pm 8.00$ & 0.300 \\
LVEF [\%] & $48.86 \pm 14.00$ & $52.29 \pm 16.32$ & $45.43 \pm 11.34$ & 0.245 \\
LZ min. dimension [mm] & $16.86 \pm 2.96$ & $15.71 \pm 2.87$ & $18.00 \pm 2.7$ & 0.235 \\
LZ max. dimension [mm] & $19.43 \pm 3.20$ & $19.14 \pm 4.02$ & $19.71 \pm 2.43$ & 0.645 \\
Ostium min. dimension [mm] & $20.93 \pm 4.78$ & $19.14 \pm 3.13$ & $22.71 \pm 5.68$ & 0.176 \\
Ostium max. dimesnion [mm] & $24.36 \pm 3.52$ & $23.29 \pm 3.50$ & $25.43 \pm 3.46$ & 0.270 \\
LAA orifice area [cm $\left.{ }^{2}\right]$ & $4.15 \pm 1.19$ & $3.94 \pm 1.20$ & $4.36 \pm 1.24$ & 0.565 \\
MV-LAA distance [mm] & $12.64 \pm 3.27$ & $12.00 \pm 3.42$ & $13.29 \pm 3.25$ & 0.515 \\
LA depth [mm] & $20.07 \pm 7.84$ & $22.14 \pm 6.12$ & $18.00 \pm 9.26$ & 0.553 \\
LAA morphology: & & & & 0.580 \\
Cauliflower & $1(7.14 \%)$ & $0(0.00 \%)$ & $1(14.29 \%)$ & \\
Chickenwing & $11(78.57 \%)$ & $6(85.71 \%)$ & $5(71.43 \%)$ & $1(14.29 \%)$ \\
Windsock & $2(14.29 \%)$ & $1(14.29 \%)$ & & \\
LAA orifice position: & $1(7.14 \%)$ & $0(0.00 \%)$ & $3(14.29 \%)$ \\
Low & $7(50.00 \%)$ & $4(57.14 \%)$ & $3(42.86 \%)$ & $3(42.86 \%)$ \\
Intermediate & $6(42.86 \%)$ & $3(42.86 \%)$ & 0.565 \\
High & & &
\end{tabular}

LA — left atrium; LAA — left atrial appendage; LAAC — left atrial appendage closure; LVEDD — left ventricular end-diastolic dimension; LVESD - left ventricular end-systolic dimension; LVEF — left ventricular ejection fraction; max. — maximum; min. - minimum; $\mathrm{MV}$ - mitral valve; LZ - landing zone

out in a 1:1 ratio. The logistic regression model was then examined to assess the quality of propensity scores. A goodness-of-fit test (Hosmer-Lemeshow) suggested good model fit $\chi^{2}=4.031(\mathrm{p}=0.909)$. Statistical significance was verified by the $\chi^{2}$ test for qualitative variables and the Mann-Whitney test for quantitative variables. A p-value $<0.05$ was considered statistically significant. The calculations were made in the XLSTAT 2021 program.

Among all patients who underwent LAAC under ICE guidance, the maximal landing zone diameter was $21.36 \pm 4.0 \mathrm{~mm}$, and the LAA depth was $24.10 \pm 7.9 \mathrm{~mm}$. The most common morphological LAA type was the chickenwing $(\mathrm{n}=17,54.84 \%)$, followed by windsock $(\mathrm{n}=10,32.26 \%)$, cactus $(\mathrm{n}=3,9.68 \%)$ and cauliflower $(\mathrm{n}=1,3.23 \%)$. Considering the position of LAA orifice, the intermediate origin was dominant $(n=15,48.39 \%)$. The high location of orifice was also often diagnosed $(\mathrm{n}=12,38.71 \%)$, while the low type was sporadic $(\mathrm{n}=4,12.9 \%)$.

The characteristics of the left atrium and the LAA in subgroups selected based on the propensity matching score are presented in Table 1.
In the ICE 3D group, the procedure duration was significantly shorter $(65.21 \pm 26.76$ vs. $84.57 \pm$ $\pm 24.13 \mathrm{~min} ; \mathrm{p}=0.005)$, and the radiation dose was significantly lower (vs. $126.15 \pm 82.28$ vs. $133.57 \pm$ $\pm 117.36 \mathrm{mGy} ; \mathrm{p}=0.038$ ), but the fluoroscopy time was only insignificantly shorter $(10.78 \pm 4.49$ vs. $12.86 \pm 8.71 \mathrm{~min} ; \mathrm{p}=0.136)$. There were no severe complications during the procedures, and they led to the effective elimination of LAA in both groups. In short-term follow-up, all patients survived, no strokes or bleeding complications were found.

The occluder position was considered optimal in $4(57.14 \%)$ cases in the ICE 3D group and $6(85.71 \%)$ in the ICE $2 \mathrm{D}$ group $(\mathrm{p}=0.237)$. Moreover, a peridevice leak was more frequent in the ICE 3D group (4 [57.14\%] vs. 1 [14.29\%]). There were no incidents of device-related thrombus. Despite the SoundStar probe's larger diameter, its use was not associated with more frequent iatrogenic atrial septal defect; $3(42.86 \%)$ cases in ICE $3 \mathrm{D}$ vs. $5(71.43 \%)$ cases in the ICE 2D group ( $p=0.28)$.

To date, results of only a few studies evaluating ICE used for imaging during LAAC have been pub- 
lished, all of them concerning guiding procedures with probes which allow obtaining $2 \mathrm{D}$ images. It was demonstrated that LAAC under ICE guidance is possible and has high technical and procedural effectiveness $[5,6]$. Nevertheless, one of the limitations of this method was the lack of $3 \mathrm{D}$ imaging. A new solution has recently emerged that enables $3 \mathrm{D}$ reconstructions based on the signal from the ICE probe.

The present study confirmed the high efficiency of LAAC performed under ICE guidance with AccuNav or SoundStar probes. The use of ICE and also $3 \mathrm{D}$ probes is not associated with a higher risk of peri-procedural complications. However, the reconstruction of $3 \mathrm{D}$ images with Soundstar is time-consuming, which contributes to the more frequent acceptance of suboptimal occluder positioning during the procedure. The study results conclude that while ICE $3 \mathrm{D}$ and $2 \mathrm{D}$ imaging allow for equally effective and safe monitoring of LAAC treatments, the use of spatial reconstructions obtained with the SoundStar transducer does not bring additional benefits. Shorter procedure times were often paid for by a suboptimal implantation effect and more frequent leakage around the occluder.

The main limitation of the presented work is the small group size in which ICE was used. Despite the validation of the propensity matching score for the very small sample size, a bias error in such cases increases and can also affect the comparison results between the two groups [7]. Also, Soundstar probes allowed only for 3D reconstruction, while real-time 3D imaging would probably impact this imaging technique's more favourable assessment.
Conflict of interest: Witold Streb, Katarzyna Mitręga and Zbigniew Kalarus are proctors of Abbott Medical.

\section{References}

1. Chow DHf, Bieliauskas G, Sawaya FJ, et al. A comparative study of different imaging modalities for successful percutaneous left atrial appendage closure. Open Heart. 2017; 4(2): e000627, doi: 10.1136/openhrt-2017-000627, indexed in Pubmed: 28761682.

2. Gafoor S, Schulz P, Heuer L, et al. Use of EchoNavigator, a novel echocardiography-fluoroscopy overlay system, for transseptal puncture and left atrial appendage occlusion. J Interv Cardiol. 2015; 28(2): 215-217, doi: 10.1111/joic.12170, indexed in Pubmed: 25676602.

3. Clemente A, Avogliero F, Berti S, et al. Multimodality imaging in preoperative assessment of left atrial appendage transcatheter occlusion with the Amplatzer Cardiac Plug. Eur Heart J Cardiovasc Imaging. 2015; 16(11): 1276-1287, doi: 10.1093/ehjci/ jev097, indexed in Pubmed: 25916628.

4. Ribeiro JM, Teixeira R, Puga L, et al. Comparison of intracardiac and transoesophageal echocardiography for guidance of percutaneous left atrial appendage occlusion: A meta-analysis. Echocardiography. 2019; 36(7): 1330-1337, doi: 10.1111/echo.14415, indexed in Pubmed: 31215709.

5. Berti S, Paradossi U, Meucci F, et al. Peri-procedural intracardiac echocardiography for left atrial appendage closure: a dual-center experience. J Am Coll Cardiol Intv. 2014; 7: 1036-1044.

6. Matsuo Y, Neuzil P, Petru J, et al. Left atrial appendage closure under intracardiac echocardiographic guidance: feasibility and comparison with transesophageal echocardiography. J Am Heart Assoc. 2016; 5(10), doi: 10.1161/JAHA.116.003695, indexed in Pubmed: 27680664.

7. Pirracchio R, Resche-Rigon M, Chevret S. Evaluation of the propensity score methods for estimating marginal odds ratios in case of small sample size. BMC Med Res Methodol. 2012; 12: 70, doi: 10.1186/1471-2288-12-70, indexed in Pubmed: 22646911. 